\title{
The History and Application of “Ashi Points"
}

\author{
Yarong Peng, Xu Du* \\ Department of Acupuncture and Massage, Shaanxi University of Traditional Chinese Medicine, Xianyang, 712046, Shaanxi Province, \\ China.
}

\author{
How to cite this paper: Yarong Peng, $\mathrm{Xu}$ \\ Du. (2021) The History and Application of \\ “Ashi Points". International Journal of \\ Clinical and Experimental Medicine \\ Research, 5(4), 485-489. \\ DOI: $10.26855 / \mathrm{ijcemr} .2021 .10 .010$ \\ Received: August 7, 2021 \\ Accepted: August 30, 2021 \\ Published: September 30, 2021 \\ *Corresponding author: $\mathrm{Xu} \mathrm{Du}, \mathrm{De}-$ \\ partment of Acupuncture and Massage, \\ Shaanxi University of Traditional Chi- \\ nese Medicine, Xianyang, 712046, \\ Shaanxi Province, China. \\ Email: dxdmzhnsh@sina.com
}

\begin{abstract}
The original meaning of "Ashi points" is an acupuncture point near or far from the affected place, which is the earliest discovered acupoint, earlier than meridian points. It became a clinical acupoint selection method in The Qin, Han, Tang and Yuan dynasties, but evolved into a strange acupoint in the Ming and Qing Dynasties, with a long history. With the extensive clinical research and application of Ashi points, it has been found that it has special therapeutic effect on various systemic diseases, especially on pain, which has been highly valued by physicians of all dynasties. However, there are still differences on the relationship between Ashi points and meridians. In this paper, the origin, location and modern clinical application of Ashi points were systematically summarized and classified, and a new classification method and treatment application criteria of Ashi points were proposed. In order to provide a new reference for the basic theory and clinical development and treatment of Ashi points.
\end{abstract}

\section{Keywords}

Ashi Points, Acupuncture, Days Should Be Hole, Indefinite Point

"Ashi points" is also known as "Tianying Point" or "Indefinite point". It has neither specific point name nor fixed location, and is mostly used for the treatment of painful sexually transmitted diseases [1]. "Ashi points" is one of the important acupoints in the development history of acupoints, which makes up for the deficiency of the fourteen meridian points and the external meridian points. It is a typical embodiment of the traditional Chinese medicine "there must be a variety of external meridian points" and occupies an important position in the clinical practice of acupuncture and moxibustion. Therefore, the in-depth study of Ashi points is of great significance to enrich the theoretical knowledge of acupoints, improve the clinical efficacy of acupuncture and moxibustion, and expand the scope of acupuncture treatment. But how to find the pain points and where the pain points come from has always been a problem.

\section{Study on the origin of "Ashi points"}

\section{1 “Taking Pain as Losing point” and “Ashi points”}

In modern times, when it comes to Ashi points, it is believed that it originated in neijing, and "taking pain as a loss" is regarded as the earliest origin of Ashi points. The word "take pain as losing” was first seen in Lingshu Jing Jin. There are 13 descriptions of "take pain as losing" in this article, which mainly discusses the circulation, onset and disease characteristics of jing Jin, and puts forward the same treatment principle for such diseases, that is, "Treat by robbing of burnt needle to know the number and take pain as losing”. The symptoms treated are mainly pain symptoms. In addition, there are no obvious pain symptoms in the original text of the disease, for this kind of disease, the treatment principles and methods are different. For diseases with obvious pain symptoms (pain, muscle 
tendon, etc.), other targeted treatments are introduced for non-painful diseases. Thus, it can be seen that in the "Neijing" "Cure in burnt needle robbery... Take pain as lose" the treatment principle is only applicable to the treatment of "tendon" and "tendon urgent" and other local pain for the performance of the disease. On the other hand, the appearance of "taking pain as infusion" every time must be after "treating burnt needle needling", so it can be seen that the acupoint selection method of "taking pain as infusion" is only applicable to the cold meridian disease treated by "burnt needle needling". It is out of context to talk about the treatment of "burnt needle robbery", regardless of the pathogenesis [2]. Thus, it can be seen that "Ashi points" can include "losing with pain", but "Ashi points" is not just "losing with pain".

\subsection{Origin and development of Ashi points}

The word "Ashi points" was first put forward by Sun Simiao, a doctor in the Tang Dynasty. In The Sixth Case of Moxibustion, "Wu Shu practiced moxibustion more often, and there is a method of Ashi. When a person has an illness, he is ordered to knead it. Moxibustion thorns are tested, so it is said that a point is also" [3]. It can be seen that Ashi points is the place where patients feel pain through clinical practice, but it is not necessarily a famous acupoint. Wang Zhuzhong, a doctor in the Southern Song Dynasty, put forward the concept of acupoints on the basis of Ashi points. "Zisheng Jing of Acupuncture and Moxibustion Back Pain" [4] said, "When I tasted it on the side of huang huang, four and a half inches of spine was removed, and the pain was dull and slight. Small moxibustion three strong, that is, no pain. It day after day even shoulder pain, but moxibustion shoulder pain. The party knows the daughter of the party is still letter cloud". Therefore, the concept of "sick place" is closely related to the "Ashi points" in "Qianjin Yaofang” [5]. In the Yuan Dynasty, Wang Guangrui named it as "indefinite point”, "Bian Que God should acupuncture yulong Jing Yulong Song Body pain” [6] said: "The whole body pain and disease is very, indefinite point should be detailed. It is believed that indefinite points are the pain points, and when acupuncture and moxibustion are used to treat the pain syndrome, the tender points of the disease should be actively sought. Ming Dynasty Wu Kun also recorded in six collections of Acupuncture Prescriptions [7]: "Indefinite point, namely qianjin Fang, 'Ashi points' ”, Jade Dragon Song said: “ 'Indefinite point' but the pain is on the left and right acupoints, lying down the needle through the pain of diarrhea, the so-called pain is lost. The Ming Dynasty Lou Ying called "Tianying point", "Compendium of Medicine Tongbi" [8]: "pain all over the body, but the needle at the pain point, regardless of meridian points, must avoid bones and muscles, the point name tianying point". "Ashi points" is regarded as the induction point of disease and is called "Tianying acupoint". Li chan in the Ming Dynasty named it as "Sanci" in Introduction to Medicine Acupuncture [9]: "People who scatter needles... Because of its disease and the needle, although the point also regardless of its or not. Refers to the pain needle pain, Xu called the day should point. This point method probably also". O hole was paid great attention in past dynasties doctor from neijing period "in pain for losing" to the Yuan dynasty Guo-rui Wang "indefinite", to the Ming dynasty li chan LouYing should point "day" and the "loose thorn" and so on, although the name is different, but similar meaning, are actually on the body surface or looking for diseases such as acid or pain reflex point or needles or abnormal moxibustion treatment, It can be found by pressing the response, and is not restricted by the location of meridians and acupoints. The acupoint selection basis is consistent with the characteristics of indications.

\section{Location of “Ashi points"}

As for the location of Ashi points, doctors of all dynasties believed that it was the place where patients felt pain, but there was no clear explanation on how to find Ashi points. So how to find Ashi points becomes a necessary problem to be solved in order to better facilitate clinical practice.

\subsection{Search for Ashi points near the affected site}

"Ashi points" is often not a specific point, so its distribution can be distributed around some points. Because its performance is pain, numbness and other discomfort, pain, numbness and other discomfort is caused by impassability of qi and blood. Acupuncture or burnt needle prick these parts to pass qi and blood, so as to achieve the purpose of treatment. If you have a headache, you can find a point in the head for treatment. As we all know, in addition to meridians, the human body also has smaller veins, such as collaterals and sun collaterals, all over the body. When the large meridians qi and blood running is not smooth, it will inevitably lead to small veins or even smaller veins running not smooth, and the most severe point of running not smooth is a point. Therefore, when you have a headache, you can find whether there is a point near the headache. If there is, you can enhance the curative effect by treating a point. 


\subsection{Ashi point was searched at the distal end of the affected site}

Ashi points can appear in any part of the body, not only confined to the vicinity of the affected part, so it should also be found in the distal end of Ashi points. The distal acupoint can be found by this meridian, by the two internal and external meridians, or by analyzing the corresponding parts of the disease cause. For example, for old and weak people with low back pain, although the low back pain point is often used for clinical treatment, but the patient's pain is severe, so baihui point of reflex point on the head is recommended for treatment [10].

\subsubsection{Search for Ashi points around meridian of affected site}

When the proximal end of the affected site is not found, it can also be searched around the meridian. Meridians are channels for qi and blood to nourish the body. When the meridians are affected by evil factors, it will inevitably affect the operation of qi and blood at other points of the meridians, resulting in the inability of qi and blood to nourish the little Sun collaterals around the big meridians, resulting in Ashi points. Abnormal reactions in certain parts of the body surface or at specific acupoints may indicate lesions in the corresponding parts, and these reaction points can help diagnose the disease. For example: The Ming Dynasty Xue Lizhai "surgery heart law" have: "in the house faint and slight pain, lung anthrax also". It is also recorded in Cheng Dan'an's Collection of Acupuncture and Moxibustion: "If you press the middle office with your hand, the pain is lung disease, and the pain and shock is lung breakage, which is difficult to cure”. For example, patients with heart disease often have tenderness points in the anterior heart area and the left shoulder and back; people with gallbladder disease may find tenderness points around the Spring. Shoulder leakage can find tenderness near the scapula and shoulder joint.

\subsubsection{Search for Ashi points around the two meridians inside the surface of the affected site}

The three Yin and three Yang meridians of hands and feet communicate with each other through meridians and collateral channels. The two meridians of the table and the inside of each other, respectively circulate in the relative position of the inner and outer limbs, and transfer at the extremities end; they also belong to the zang-fu organs, which form the relationship between the zang-fu organs and the Yin and Yang. The relationship between the 12 meridians on the outside and inside not only strengthens the connection between the two meridians on the outside and inside of each other, but also makes one viscera and one viscera on the outside and inside of each other cooperate with each other in physiological function and influence each other in pathology. In the treatment, the acupoints on the two meridians inside and outside each other often cross. Therefore, you can also search for a hole around the two meridians.

\subsubsection{The corresponding position of the cause of the disease should be searched for Ashi points}

Huangdi Neijing says: "What is inside must be outside". "Ashi points” is not only the reaction caused by poor meridians, but also the reaction caused by the dysfunction of zang-fu organs and qi and blood (zongqi, Yingwei's qi) and sanjiao. Therefore, in the analysis of the disease to find the relevant viscera, viscera corresponding to different parts of the vicinity may also find Ashi points.

\section{Causes, classification and treatment of Ashi points}

\subsection{Causes of Ashi points}

Ashi point is the discomfort of pain, acid, numbness and so on due to the poor operation of qi and blood in its parts. Therefore, its cause should be studied from the point of focus of qi and blood not running smoothly. There are two reasons for the poor operation of qi and blood: one is qi and blood deficiency, the other is qi and blood stagnation. Qi and blood deficiency results in tendons that cannot be nurtured by blood or promoted by qi. The stagnation of qi and blood causes the blood to move unsmoothly and to choke somewhere. Through acupuncture and moxibustion, Ashi points can supplement its qi and blood by supplementing method, and can also pass its stagnation by purging method, so that qi can heal.

\subsection{Classification and treatment of Ashi points}

The performance of a point is not only pain, acid, hemp, swelling and other places are also a point. Even the skin is particularly easy to bad can also be considered as an acupoint. In addition, some Ashi points after pressing the discomfort relieved, some after pressing the discomfort increased. Due to their different performance, they are classified as follows:

\subsubsection{Ashi points of qi and blood deficiency}

Ashi points of qi and blood deficiency usually shows that temperature is reduced and pressure is mild. Often due 
to cold guest meridians lead to qi and blood running not smooth cannot warm, treatment should be moxibustion, tonic method, etc. "Precious Treasure Important Prescription Moxibustion Examples" said, "Every hole in the body... With muscle texture, joints, and hands, the patient quickly, so careful and serene heart, you can get the ear". It can be seen that "muscle texture", "joint solution", "seam meeting” and "Wan Depression" can all be used as the location of Ashi points. "If it is in the place, regardless of the hole, it is fast or painful, that is, yun Ashi points", and knowing comfort is also the basis of Ashi points selection. "Neijing" also has this kind of record, "Lingshu five evil" cloud: "evil in the lung, the disease skin pain... On the back of the three sections of the five zang organs, press it with the hand, quickly and quickly, but it is stabbing", that is, to find the patient feel comfortable by pressing the part of the operation. This kind of acupoint is an acupoint of qi and blood deficiency. "Su Ask Lift pain" has "warm feeling" as the basis for the selection of AShi points: "Cold guest in the back of yu's pulse is pulse weep, pulse weep is blood deficiency, blood deficiency is pain, the shu note in the heart, so the pain, according to the heat to the heat to the pain". That is, the surface of the body with a special sense of warmth as a point for treatment. Cold guest meridians can also change the tendons. "Su Ask bone empty theory" said: "the lack of pelvic bone cut on the hard pain such as tendon moxibustion", Wang Bing note: "the lack of pelvic bone cut on the hard pain such as tendon, by the lack of its name, when all the moxibustion". Therefore, "the strong pain of cutting on the basin is like tendon" refers to looking for obvious cable nodules in the basin, which is used as the surgical site of moxibustion.

\subsubsection{Ashi points of Qi and blood stagnation}

Qi and blood stagnation of Ashi points is usually shown as severe tenderness, temperature and other symptoms. Often because of qi and blood stasis, treatment should be acupuncture, diarrhea, etc.

Ashi points are widely used in combination with other meridian points as acupuncture prescriptions. In Mianxuetang Acupuncture Integration Jiancheng by Liao Runhong in the Qing Dynasty [11], there are: "Xiang Qiang: Fengmen, Shoulder well, Fengchi, Kunlun, Tianzhu, Fengfu and Juangu. Refer to their meridians for treatment and also needle Ashi points. The method of following the needle along with the pain is detailed in the sore part of the arm. If it can be done, it will be all magic. It can be seen that when Liao Runhong selected acupoints, in addition to the 14 acupuncture points, he especially included Ashi points in the acupuncture prescription, which proved to be effective. In clinical diagnosis and treatment, Wang Zhizhong emphasized to find the "pain" related to diseases and syndromes by pressing, that is, the site of acupuncture and moxibustion. Just as in the treatment of knee pain by moxibustion Zisheng Jing Knee pain, moxibustion is used to treat knee pain: "But according to the point of pain, that is, the affected place, moxibustion is not detained. Wang believes that the pain point is the disease, acupuncture and moxibustion, the curative effect is significant [12].

\subsection{The Prognosis}

Zhan Yongkang in "acupuncture and moxibustion acupoint tenderness diagnosis and treatment relationship" mentioned: “a spermatorrhea patient, transverse bone tenderness, the first stab sanyinjiao, Shenshu, jinggong, Guan Yuan, needle after the transverse bone tenderness did not disappear and spermatorrhea stopped, the patient will stop acupuncture, but not to January and recurrence... Three times, the transverse bone tenderness completely disappeared, spermatorrhea self-thorn no longer recurrence". It can be seen from the above cases that sometimes other symptoms disappear, but Ashi points still has pathological reaction, which means that the disease has not been completely treated, and the condition may be repeated. It can be seen that the disappearance of tenderness points is also a sign to measure whether the disease is really cured [13].

\section{Summary}

$\mathrm{O}$ is the study of the cavity has a long history, clinical, the hole is a wide range of indications, main treatment due to qi and blood block impassability disease of zang-fu organs and meridians, through regulating qi and blood, dredge main and collateral channels, to achieve the purpose of general principles of pain, has obtained the remarkable curative effect in the treatment, make up for the deficiency of the fourteen meridian and the singular point, embodies the holistic concept of traditional Chinese medicine. There is no clear cognition about the occurrence of Ashi points. This article is only my hypothesis about the position of Ashi points, and the specific research needs to be explored by future generations.

\section{References}

[1] Cheng Xinnong. (1999). Chinese Acupuncture and Moxibustion Science [M]. Beijing: People’s Medical Publishing House, 1999: 120 . 
[2] Liu wenhao. (2020). Analysis of the original meaning of "pain as losing” and ashi point [J]. Massage and Rehabilitation Medicine, 2020, 11(22): 22-23.

[3] Tang Sun Simiao. (2011). Beijing: China Medical Science and Technology Press, 2011: 510-511.

[4] Wang Zhuzhong. (2007). Zisheng Jing of acupuncture and moxibustion [M]. Beijing: People’s Medical Publishing House, 2007: 11.

[5] Yu Bing, Wang Cong, Zhang Yongchen, Jia Hongling. (2016). Discussion on ashi point [J]. Clinical Journal of Acupuncture and Moxibustion, 2016, 32(03): 83-85.

[6] Huang Longxiang. (1996). Integration of Acupuncture and Moxibustion Classics [M]. Beijing: Huaxia Press, 1996.

[7] Wu Kun. (1991). Interpretation of The Sixth set of Needle [M]. Beijing: China Medical Science and Technology Press, 1991: 140.

[8] Lou Ying. (1996). Compendium of Medical Science [M]. Beijing: China Traditional Chinese Medicine Publishing House, 1996: 210.

[9] Li Chan. (1995). Introduction to Medical Science [M]. Beijing: China Traditional Chinese Medicine Publishing House, 1995: 107-108.

[10] Wang Feng, Xiao Bing, Huang Mei, Zhao Rong. (2015). Journal of Anhui University of Traditional Chinese Medicine, 2015, 34(06): 54-56.

[11] Liao Runhong. (1994). Mian Xuetang Acupuncture integration [M]. Beijing: China Traditional Chinese Medicine Publishing House, 1994: 62.

[12] Gan Jianxing. (1993). Discussion on a shi point and its clinical application [J]. Chinese Folk Therapeutics, 1993, 3(9): 7-8.

[13] Shao Shuijin, Xie Qiang. (1999). Wei Jia acupuncture experience collection [M]. Shanghai: Shanghai University of Traditional Chinese Medicine Press, 1999: 56. 